Internat. J. Math. \& Math. Sci.

Vol. 24, No. 1 (2000) 59-61

S0161171200003537

(c) Hindawi Publishing Corp.

\title{
ON AN ISOLATION AND A GENERALIZATION OF HÖLDER'S INEQUALITY
}

\section{XIAOJING YANG}

(Received 23 December 1998 and in revised form 16 June 1999)

ABSTRACT. We generalize the well-known Hölder inequality and give a condition at which the equality holds.

Keywords and phrases. Hölder inequality, equality.

2000 Mathematics Subject Classification. Primary 34C10; Secondary 34L05.

Let us consider the famous Hölder inequality [1]

$$
\sum_{i=1}^{m} \prod_{j=1}^{n} X_{i j} \leq \prod_{j=1}^{n}\left(\sum_{i=1}^{m} X_{i j}^{p j}\right)^{1 / p j},
$$

where $X_{i j}>0(1 \leq i \leq m, 1 \leq j \leq n), p_{j}>1, \sum_{j=1}^{n}\left(1 / p_{j}\right)=1$.

We give a generalization of (1), first, we need the following lemmas.

LEMMA 1. Let $y_{i}=\prod_{j=1}^{n} X_{i j}, i=1,2, \ldots, m$, then $\sum_{k=1}^{n} 1 / p_{k}\left(\sum_{i=1}^{m} y_{i} \ln z_{i k}\right)=0$, where $z_{i k}=X_{i k}^{p k} / y_{i}, i=1,2, \ldots, m$.

Proof. Since $y_{i}=\prod_{j=1}^{n} X_{i j}$, we have $\ln y_{i}=\sum_{j=i}^{n} \ln X_{i j}$ and

$$
\begin{aligned}
\sum_{k=1}^{n} \frac{1}{p_{k}} \ln z_{i k} & =\sum_{k=1}^{n} \frac{1}{p_{k}} \ln \left(\frac{X_{i k}^{p k}}{y_{i}}\right)=\sum_{k=1}^{n} \frac{1}{p_{k}}\left(p_{k} \ln X_{i k}-\ln y_{i}\right) \\
& =\sum_{k=1}^{n} \ln X_{i k}-\sum_{k=1}^{n} \frac{1}{p_{k}} \ln y_{i}=\ln \prod_{k=1}^{n} X_{i k}-\ln y_{i}=0, \quad i=1,2, \ldots, m
\end{aligned}
$$

here we have used the fact $\sum_{k=1}^{n}\left(1 / p_{k}\right)=1$, from the above calculations we obtain

$$
\left(\sum_{i=1}^{m} y_{i}\right)\left(\sum_{k=1}^{n} \frac{1}{p_{k}} \ln z_{i k}\right)=\sum_{k=1}^{n} \frac{1}{p_{k}}\left(\sum_{i=1}^{m} y_{i} \ln z_{i k}\right)=0 .
$$

LEMMA 2. If $a, b$ are positive numbers, then

$$
\begin{aligned}
& (\ln a-\ln b)\left(a^{t}-b^{t}\right) \geq 0, \quad \text { if } t \geq 0, \\
& (\ln a-\ln b)\left(a^{t}-b^{t}\right) \leq 0, \quad \text { if } t \leq 0,
\end{aligned}
$$

and the equalities hold if and only if $(a-b) t=0$. 
Proof. If $a=b$, (4) holds, let $a \neq b$, by the mean value theorem $f(a)-f(b)=$ $(a-b) f^{\prime}(z)$ with $z \in(a, b)$, we have

$$
\begin{aligned}
& \ln a-\ln b=(a-b) \frac{1}{z_{1}}, \quad z_{1} \in(a, b), \\
& \left(a^{t}-b^{t}\right)=(a-b) t z_{2}^{t-1}, \quad z_{2} \in(a, b)
\end{aligned}
$$

hence

$$
(\ln a-\ln b)\left(a^{t}-b^{t}\right)=\frac{(a-b)^{2} t z_{2}^{t-1}}{z_{1}} \begin{cases}\geq 0, & \text { if } t \geq 0 \\ \leq 0, & \text { if } t \leq 0\end{cases}
$$

and the equality holds if and only if $t=0$.

The main result of our paper is the following theorem.

THEOREM 3. If $X_{i j}>0(1 \leq i \leq m, 1 \leq j \leq n), p_{i}>1, \sum_{i=1}^{n}\left(1 / p_{i}\right)=1$, let

$$
h(t)=\prod_{k=1}^{n}\left[\sum_{i=1}^{m} y_{i}\left(\frac{X_{i k}^{p_{k}}}{y_{i}}\right)^{t}\right]^{1 / p_{k}}=\prod_{k=1}^{n}\left[\sum_{i=1}^{m}\left(\prod_{j=1}^{n} X_{i j}\right)^{(1-t)}\left(X_{i k}^{p_{k}}\right)^{t}\right]^{1 / p_{k}} .
$$

Then

$$
\begin{aligned}
& h^{\prime}(t) \geq 0, \quad \text { if } t \geq 0, \\
& h^{\prime}(t) \leq 0, \quad \text { if } t \leq 0,
\end{aligned}
$$

the equality holds if and only if $t=0$, or $X_{i k}^{p_{k}} / \prod_{\tau=1}^{n} X_{i \tau}=X_{j k}^{p_{k}} / \prod_{\tau=1}^{n} X_{j \tau}$ for $1 \leq i, j \leq m$, $k=1,2, \ldots, n$, that is, $X_{1 k}^{p_{k}} / \prod_{j=1}^{n} X_{1 j}=X_{2 k}^{p_{k}} / \prod_{j=1}^{n} X_{2 j}=\cdots=X_{m k}^{p_{k}} / \prod_{j=1}^{n} X_{m j}$.

Proof. Let $H(t)=\ln h(t), t \in \mathbb{R}$, then by Lemmas 1 and 2 we obtain

$$
\begin{aligned}
H^{\prime}(t) & =\frac{h^{\prime}(t)}{h(t)}=\sum_{k=1}^{n} \frac{1}{p_{k}} \frac{\left(\sum_{i=1}^{m} y_{i} z_{i k}^{t} \ln z_{i k}\right)}{\left(\sum_{i=1}^{m} y_{i} z_{i k}^{t}\right)} \\
& =\sum_{k=1}^{n} \frac{1}{p_{k}} \frac{\left(\sum_{i=1}^{m} y_{i} z_{i k}^{t} \ln z_{i k}\right)}{\left(\sum_{i=1}^{m} y_{i} z_{i k}^{t}\right)}-\sum_{k=1}^{n} \frac{1}{p_{k}} \frac{\left(\sum_{i=1}^{m} y_{i} \ln z_{i k}\right)}{\left(\sum_{i=1}^{m} y_{i}\right)} \\
& =\sum_{k=1}^{n} \frac{1}{p_{k}} \frac{\sum_{1 \leq i<j \leq m} y_{i} y_{j}\left(\ln z_{i k}-\ln _{j k}\right)\left(z_{i k}^{t}-z_{j k}^{t}\right)}{\left(\sum_{i=1}^{m} y_{i}\right)\left(\sum_{i=1}^{m} y_{i} z_{i k}^{t}\right)} \begin{cases}\geq 0, & \text { if } t \geq 0, \\
\leq 0, & \text { if } t \leq 0 .\end{cases}
\end{aligned}
$$

The equality holds if and only if $t=0$ or

$$
z_{1 k}=z_{2 k}=\cdots=z_{m k}, \quad k=1,2, \ldots, n,
$$

where $y_{i}=\prod_{j=1}^{n} X_{i j}, z_{i k}=X_{i k}^{p_{k}} / \prod_{j=1}^{n} X_{i j}, i=1,2, \ldots, m, k=1,2, \ldots, n$. This completes the proof .

COROLlaRY 4. Let $t_{2}<t_{1} \leq 0$ or $0 \leq t_{1}<t_{2}$, we have

$$
\prod_{k=1}^{n}\left[\sum_{i=1}^{m}\left(\prod_{j=1}^{n} X_{i j}\right)^{1-t_{1}} X_{i k}^{p_{k} t_{1}}\right]^{1 / p_{k}} \leq \prod_{k=1}^{n}\left[\sum_{i=1}^{m}\left(\prod_{j=1}^{n} X_{i j}\right)^{1-t_{2}} X_{i k}^{p_{k} t_{2}}\right]^{1 / p_{k}},
$$


equality holds if and only if for any $k=1,2, \ldots, n$,

$$
\frac{X_{i k}^{p_{k}}}{\prod_{\tau=1}^{n} X_{i \tau}}=\frac{X_{j k}^{p_{k}}}{\prod_{\tau=1}^{n} X_{j \tau}}, \quad \text { for } 1 \leq i \leq j \leq m,
$$

and for all $0<t<1$,

$$
\sum_{i=1}^{m} \prod_{j=1}^{n} X_{i j} \leq \prod_{k=1}^{n}\left[\sum_{i=1}^{m}\left(\prod_{j=1}^{i} X_{i j}\right)^{1-t} X_{i k}^{p_{k} t}\right]^{1 / p_{k}} \leq \prod_{i=1}^{n}\left(\sum_{j=1}^{m} X_{i j}^{p_{j}}\right)^{1 / p_{j}}
$$

equality holds only (2') holds.

\section{REFERENCES}

[1] E. F. Beckenbach and R. Bellman, Inequalities, Springer-Verlag, Berlin, 1961. MR 28\#1266. Zbl 097.26502.

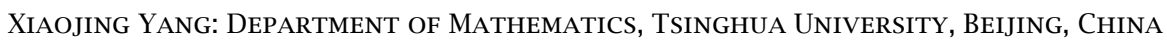




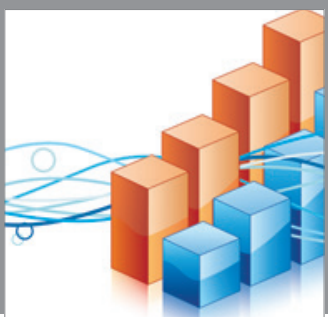

Advances in

Operations Research

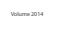

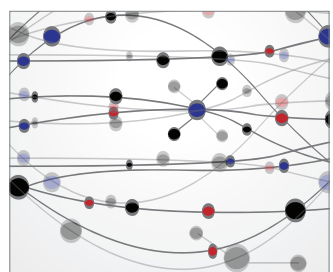

\section{The Scientific} World Journal
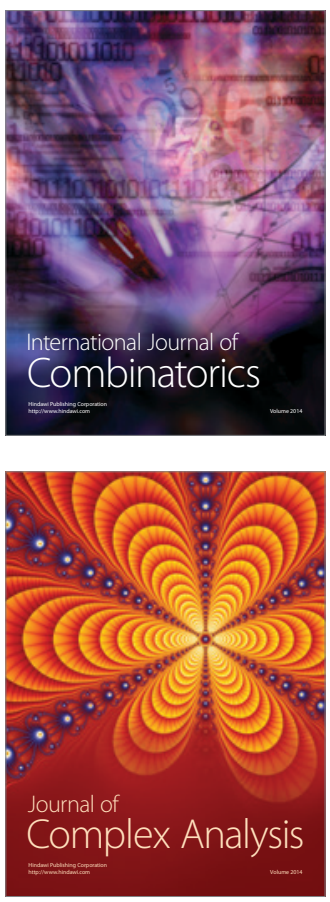

International Journal of

Mathematics and

Mathematical

Sciences
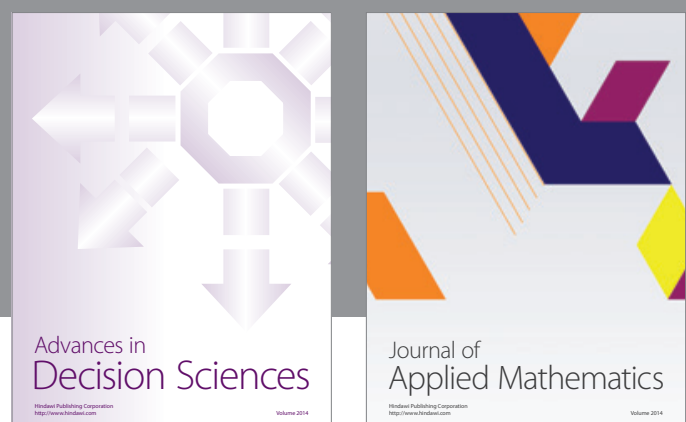

Journal of

Applied Mathematics
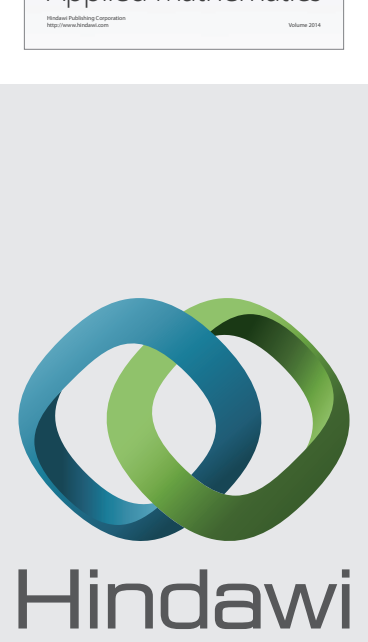

Submit your manuscripts at http://www.hindawi.com
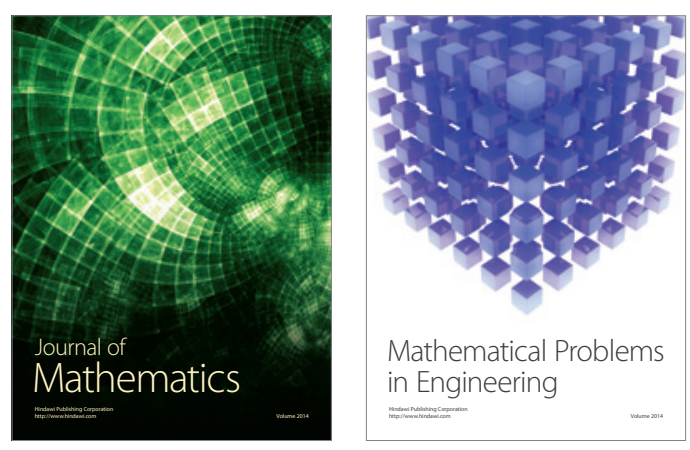

Mathematical Problems in Engineering
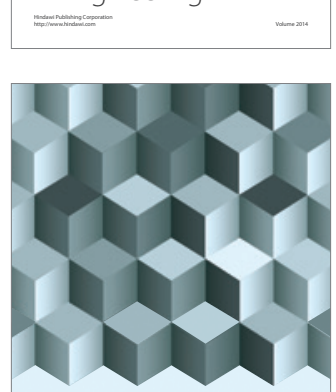

Journal of

Function Spaces
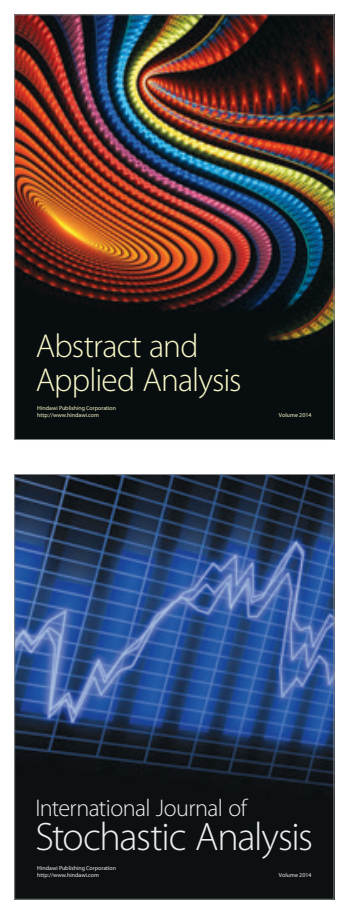

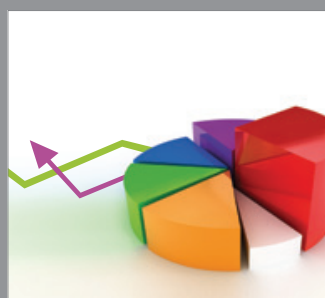

ournal of

Probability and Statistics

Promensencen
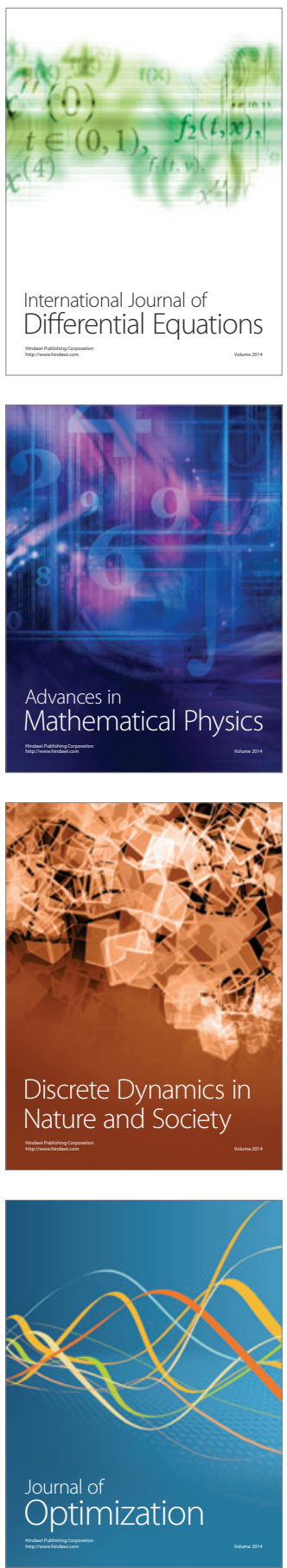